\title{
Computational prediction and experimental validation of a novel synthesized pan-PIM inhibitor PI003 and its apoptosis-inducing mechanisms in cervical cancer
}

\author{
Zhongyu Liu ${ }^{1, *}$, Weihua $\mathrm{He}^{1,{ }^{*}}$, Jianglin $\mathrm{GaO}^{1}$, Junhua $\mathrm{Luo}^{2}$, Xian Huang ${ }^{1}$, \\ Chunfang Gao $^{1}$ \\ ${ }^{1}$ Anal-Colorectal Surgery Institute, No.150 Central Hospital of PLA, Luoyang, Henan 471031, China \\ ${ }^{2}$ Department of Obstetrics \& Gynecology, No.150 Central Hospital of PLA, Luoyang, Henan 471031, China \\ *These authors have contributed equally to this work \\ Correspondence to: \\ Chunfang Gao, e-mail: gaochunfang2010@163.com \\ Keywords: PIM kinase family, Pan-PIM inhibitor (PI003), HeLa cell, Apoptosis, MicroRNA \\ Received: December 20, $2014 \quad$ Accepted: January 10, $2015 \quad$ Published: February 04, 2015
}

\section{ABSTRACT}

PIM protein family, short-lived serine/threonine kinases (PIM1, PIM2 and PIM3), are weak oncogenes but contribute to tumorigenesis as cancer targets. Thus, design of a novel pan-PIM inhibitor is still a challenge for current cancer drug discovery. Herein, we used a Naïve Bayesian model to construct the PIM network and identified Bad and Hsp90 to interact with PIMs. Then, we screened a series of candidate small-molecule compounds targeting PIMs, and subsequently synthesized a novel small-molecule compound PI003 with remarkable anti-proliferative activities in cervical cancer cells. Moreover, we found that PI003 induced apoptosis via the death-receptor and mitochondrial pathways by targeting PIMs and affecting Bad and Hsp90. Combined with microRNA microarray analyses, we demonstrated that some microRNAs such as miR-1296 and miR-1299 could affect PIM1-STAT3 pathway in PI003-induced apoptosis. Finally, we reported that PI003 had remarkable anti-tumor activity and apoptosis-inducing effect in in vivo mouse model. In conclusion, these results demonstrate that PI003, as a novel synthesized pan-PIM inhibitor, induces the death-receptor and mitochondrial apoptosis involved in microRNA regulation, and also possessed remarkable anti-tumor activity and apoptosis-inducing effect in vivo. Thus, these findings would shed light on discovering more potential new small-molecule pan-PIM inhibitors in future cervical cancer therapy.

\section{INTRODUCTION}

PIM proteins are a family of short-lived serine/ threonine kinases that are highly evolutionarily conserved in multicellular organisms [1]. PIM kinase family is composed of three members, PIM1, PIM2, and PIM3, which are highly homologous at the amino acid level (PIM1 and PIM3 are 71\% identical, whereas PIM1 and PIM2 share $61 \%$ homology), but differ partially in their tissue distributions [2]. Unlike other kinase activities, the activity of PIM kinases is not primarily regulated by phosphorylation; instead, PIM kinases are mainly regulated by transcription. And, PIM kinases do not have a regulatory domain and are constitutively active when expressed [3]. Thus, PIM kinases can appear to be regulated at the level of transcription, translation, and even proteasomal degradation [4].

PIM family members are weak oncogenes but can contribute to tumorigenesis by selectively enhancing tumorigenic capabilities [5]. This effect appears to depend on the tissue and the nature of the pathways activated by the molecularly cooperating oncogenes [6]. PIM kinases represent interesting drug targets because they are often overexpressed in many cancers and are involved in cancer-specific pathways, such as cell survival, cell cycle progression and cell migration. For instance, blocking PIM1 function via the introduction of a dominant-negative PIM1 sensitizes pancreatic cancer cells to apoptosis induced by glucose deprivation. Moreover, dominantnegative PIM1 reduces tumorigenicity in pancreatic 
cancer cells and HeLa xenograft mouse models [7, 8]. The emerging importance of PIM kinases in human tumorigenesis has increased interest in developing small molecule inhibitors targeting these proteins. Different classes of PIM inhibitors have recently been reported [9-11], but only a few of them have been tested in cellbased assays or animal models to demonstrate their anticancer activities. In addition, only a few of these inhibitors are effective against all PIM family kinases because most of them have been focused on PIM1 [12, 13]. Due to functional redundancy, simultaneous targeting of all PIM kinases can be advantageous in treating cancer patients. Thus, it will led to the design and synthesis of more new pan-PIM inhibitors for current and future cancer therapy.

In this study, we reported herein that PI003, as a novel synthesized pan-PIM inhibitor, could induce the death-receptor and mitochondrial apoptosis involved in some microRNA regulation, and also possessed remarkable anti-tumor activity and apoptosis-inducing effect in vivo.

\section{RESULTS}

\section{In silico analysis of the PIM kinase family network}

Four heterogeneous types of evidence were integrated and the likelihood ratios were used as the reliability of individual dataset to infer PPIs by using the Naïve Bayesian theorem (Figure 1A). We then used the ROC curve to evaluate the performances of predictions. A protein pair is predicted to be positive when its likelihood ratio is above a particular cutoff, to be negative otherwise (Figure 1B). To get an appropriate composite LR cutoff, we plot the ratio of true positive to false positive (TP/FP) as the function of the cutoff of likelihood ratio. We further modified the globe human PPI into the PIM kinases PPI (Figure 1C). 36 proteins were predicted to interact with PIM1. Such as BMX, CDNIA, MDM2, STAT3 and PTPA are all apoptotic proteins. 14 proteins interacted with PIM2, like apoptotic protein NEMO and all PIM kinases interacted with H90SB and BAD, which were involved in apoptosis. BAD, affecting the level of heterodimerization of $\mathrm{Bcl}-\mathrm{X}(\mathrm{L}), \mathrm{Bcl}-2$ and $\mathrm{Bcl}-\mathrm{W}$ with BAX, were reported been suppressed Ser112 phosphorylation by PIM kinases. The results showed the creditability of our network. And the PPI network was built for further research of miRNAPIM-other gene/protein pathways.

\section{Candidate PIM inhibitor screening and chemical synthesis of PI003}

The candidate drugs were carried out by molecule docking, after docking screening the FDA-approved small molecule compounds, 200 top-scored drugs are selected out. From them we chose 20 drugs for further experimentally screening, which were P1-P20 (Supplementary Table S1). P1-P20 are varies from each other in the structure, that means the structural diversity is considered. The MTT assay for P1-P20 showed that P1, P4, P9 and P20 had remarkable inhibitory effects of HeLa cells. Then, we selected out the best one, P9 (Chlorpromazine) (Drugbank number: DB00477) for its best inhibitory effect and capability for further chemical modifications. (Figure 1D).

The synthesis of compound PI003 was shown (Figure 2). 5-(benzyloxy)-2-iodophenol was treated with 4-(benzyloxy)-1-fluoro-2-nitrobenzene and then the reaction was triggered by $\mathrm{K} 2 \mathrm{CO} 3$ to obtain the intermediate 4-(benzyloxy)-1-(5-(benzyloxy)-2iodophenoxy)-2-nitrobenzene in a total yield of $68 \%$. After hydrogenation catalyzed by the $\mathrm{Fe} / \mathrm{HCl}$ (yield almost $100 \%$ without additional purification), the nitro group was reduced into amino. The intermediate 5-(benzyloxy)2-(5-(benzyloxy)-2-iodophenoxy) aniline was further reacted by $\mathrm{K} 2 \mathrm{CO} 3$ and DMEDA to afford the product 2 , 8-bis(benzyloxy)-10H-phenoxazine (yield 79\%). Then benzyl 4-chlorobutanoate was added to the reaction mixture in present of $\mathrm{K} 2 \mathrm{CO} 3$, after final hydrogenation catalyzed by the $\mathrm{H} 2$ and $\mathrm{Pd}-\mathrm{C}$ (yield almost $100 \%$ ), the compound PI003 was obtained. The high degree of symmetry in these molecules enabled facile confirmation by NMR techniques. The purity of all compounds was above $97.0 \%$ determined by HPLC normalization method. Furthermore, the structures of these compounds were further verified by ESI-MS. All the spectra displayed a very prominent peak corresponding to the compounds complexed with protons or sodium cation. PI003: ${ }^{1} \mathrm{H}$ NMR (400 MHz, $\left.\mathrm{CDCl}_{3}\right) \delta 6.86-6.66(\mathrm{~m}, 2 \mathrm{H}), 6.63$ $(\mathrm{d}, J=2.4 \mathrm{~Hz}, 1 \mathrm{H}), 4.61(\mathrm{~s}, 1 \mathrm{H}), 3.93(\mathrm{t}, J=11.2 \mathrm{~Hz}, 1$ H), 2.30 (t, $J=11.2 \mathrm{~Hz}, 1 \mathrm{H}), 1.89$ (t, $J=11.3 \mathrm{~Hz}, 1 \mathrm{H})$. ${ }^{13} \mathrm{C}$ NMR $\left(100 \mathrm{MHz}, \mathrm{CDCl}_{3}\right) \delta 177.25,153.29,140.19$, $133.58,119.19,113.45,105.28,49.05,30.97,23.77 . \mathrm{m} / \mathrm{z}$ $=324.0851(\mathrm{M}+\mathrm{Na})$.

\section{Molecular docking and MD simulations of PIM kinases/PI003 complex}

PI003 binds to the PIM kinases much better than P9 (Figure 3A). Of note, PIM1, PIM2 and PIM3 are highly homologous at the amino acid level. There is a long and narrow gap in the deep of the active pocket, which is the key side of PIM kinases. PI003 as a "tail" with carboxyl having Hydrogen bonding in the gap. To increase the stability, two hydroxies are added to binding with oxygen atoms outside of the gap. The hydroxies act like the key to the active pocket that brings the good result of MD simulations. As a pan-PIM inhibitor, the docking showed that PI003 target PIM1 better and it can binding to PIM2 and PIM3. 

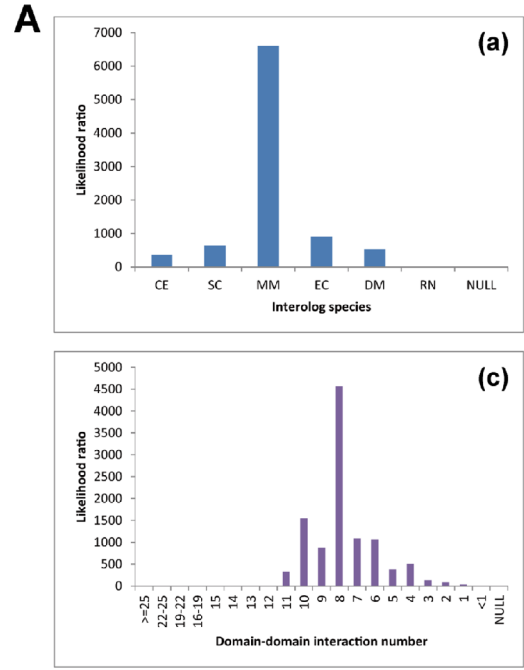

B

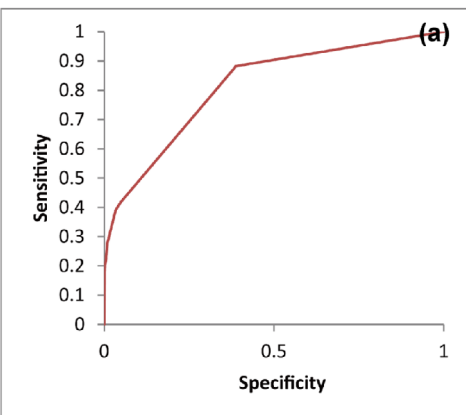

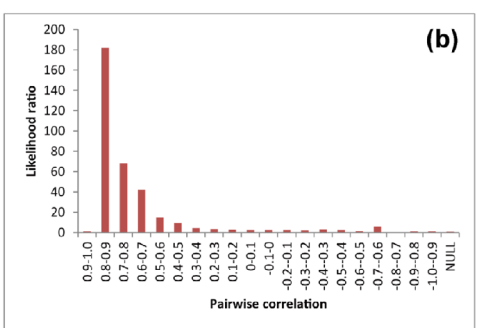
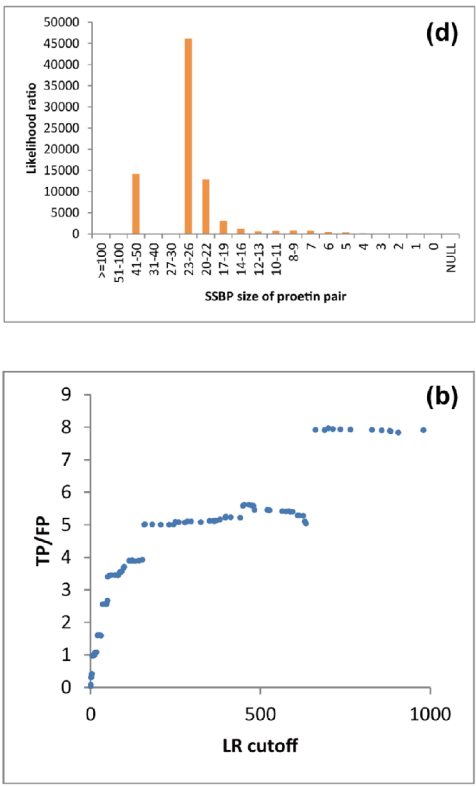

C
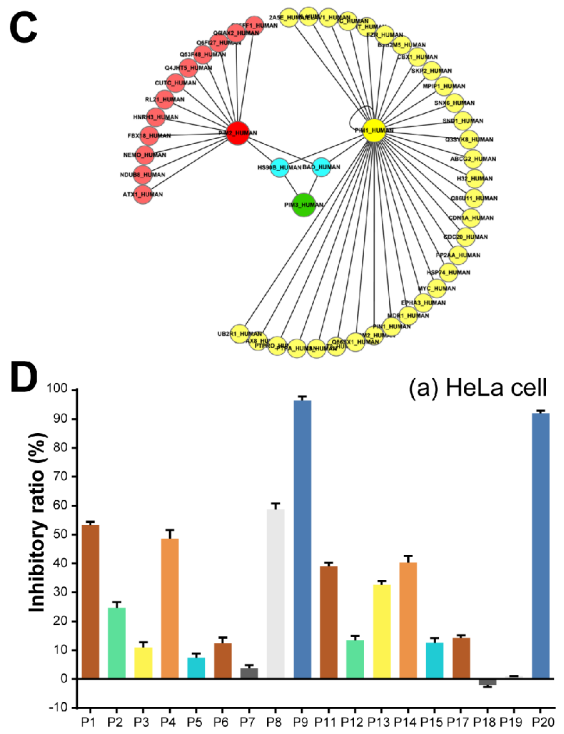

(b) C4-I cell

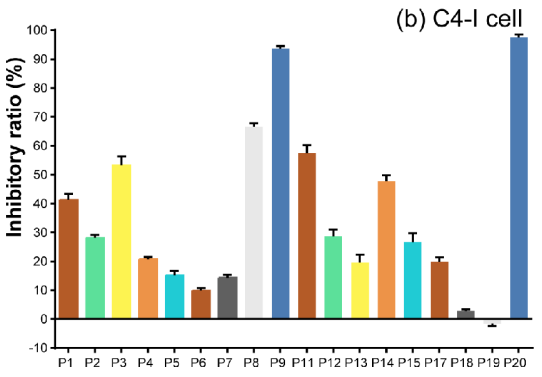

Figure 1: Network-based identification of PIM-modulated apoptotic pathways and screening of candidate PIM inhibitors. (A) Four heterogeneous types of evidence were integrated and calculated as the likelihood ratios. (B) ROC curves for evaluating the performances. NB-Loc and NB-Ran denote the Naïve Bayesian model that integrate all the evidence sources and are based on the negative set Loc-NRS and Ran-NRS, respectively. The prediction model is based on the negative reference set Loc-NRS except the NB-Ran model that uses Ran-NRS as the negative reference set. TP/FP ratios (true positive versus false positive) are calculated at different LR cutoffs. (C) The PPI of PIM kinases. (D) The MTT assay of candidate compounds.

As shown in Figure 3B, the MD simulations were carried out for $10 \mathrm{~ns}$ for the three inhibitor-PIM complexes. The RMSD values of PIM1, PIM2 and PIM3 backbone atoms involving the initial minimized structure, which plotted in Figure 3B, were calculated through the phase of the simulation to evaluate the reliable stability of the MD trajectories and the difference of the stabilities in the MD simulations. All of inhibitorPIM complexes were found to reach equilibrium after $1 \mathrm{~ns}$ of the simulation phase. As showed in Figure 3, the RMSD values read 1.2, 1.4 and $1.3 \AA$ for the PIM1, PIM2 and PIM3-inhibitor complexes, respectively, with lower than $0.5 \AA$ deviation. The study demonstrated that the trajectories of the MD simulations were stable after $1 \mathrm{~ns}$ for the three complexes, then the binding free energy calculation and free energy decomposition could be carried out on base of the snapshots extracted from 1 to $10 \mathrm{~ns}$.

The binding free energies had been calculated through the MM/GBSA method with the aid of the single trajectory protocol. For analysis of the binding free energy, the 450 snapshots were extracted at a time interval of 20 ps from the 1 to $10 \mathrm{~ns}$ of MD trajectories. The calculated binding free energies and components were shown (Table 1). It showed that the binding free energies of PIM1, PIM2 and PIM3-inhibitor complexes were $-43.69,-38.81$ and $-42.12 \mathrm{kcal} / \mathrm{mol}$, respectively. From Table 1, the intermolecular van der Waals and the electrostatics interactions both play an important role in binding, but polar salvation terms act oppositely. Nonpolar solvation terms, corresponding to the burial of SASA upon binding, are moderately favorable. In order to further investigate the influence of the configuration on the hydrogen bonding network, the visible percentage of hydrogen bonds during the MD simulations was calculated and the results was displayed (Table 1 and Table 2). The inhibitory ratio of HeLa and C4-I cells treated with PI003 and P9 were carried out by MTT analysis and the effect of PI003 gained remarkably both in HeLa and C4-I cells (Figure 3C). The IC50 of PI003 were $3.23 \mu \mathrm{M}$ and 5.38 $\mu \mathrm{M}$ in HeLa and C4-I cells, respectively. And the IC50 of P9 were $6.34 \mu \mathrm{M}$ and $10.97 \mu \mathrm{M}$. The inhibitory ratio of HeLa cells is higher than C4-I cells, thereby HeLa cells were selected for further studies. 

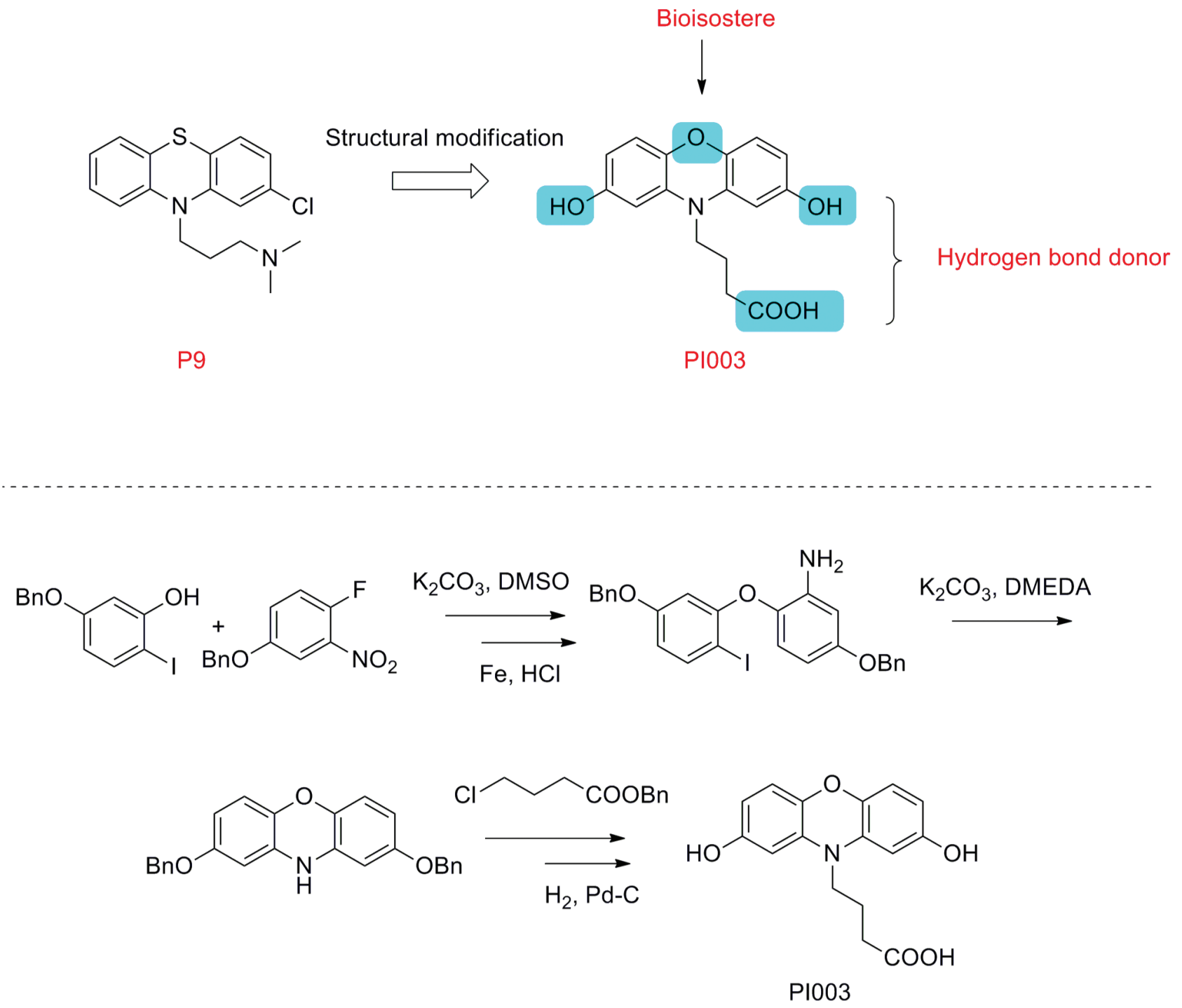

Figure 2: Chemical structure modification strategies of PI003. Compound PI003 was obtained by multi-steps chemical synthesis. The high degree of symmetry in these molecules enabled facile confirmation by NMR techniques.

\section{PI003 induces apoptosis via the death-receptor and mitochondrial pathways in HeLa cells}

Meanwhile, typical characteristics of apoptosis were also observed in PI02-treated HeLa cells. These apoptotic characteristic changes included chromatin condensation and margination at the nuclear periphery (Figure 4A). Hoechst 33342 staining also showed a higher degree of apoptosis in the HeLa cells created with PI003 compared with the negative control (Figure 4B). When PI003 was added, the change of subcellular localization of phosphatidylserine was observed under fluorescence microscope, which is a typical apoptotic feature (Figure 4C). With remarkable reduce of DNA content, the percentage increasing of HeLa cells death also measured by flow cytometry (Figure 4D). Under the microscope, typical apoptotic manifestation are observed.
Moreover, we examined PI003 induced HeLa cell apoptosis via both death-receptor and mitochondrial pathways by western blot analysis (Figure 5). After treatment with PI003, the levels of Fas, Fas L and FADD were increased. For caspase family, pro-caspase 8 and pro-caspase 3 were decreased, whereas the levels of caspase 8 and caspase 3 were increased. PARP, substrate of caspase 3, was observed a time-dependent cleaving in PI003-treated HeLa cells. Then, we investigated the involvements of Bax, pro-caspase 9 and caspase 9 in PI003-induced apoptosis. These results suggest that PI003 induces HeLa cell apoptosis via the death-receptor and mitochondrial pathways. Interestingly, in the good agreement with above-mentioned prediction (Figure 1C), we found that $\mathrm{p}$-Bad and Hsp90 were both decreased in PI003-treated HeLa cells, indicating that PI003 may be targeting PIM1/2/3-p-Bad/Hsp30 pathway in cancer cells. 

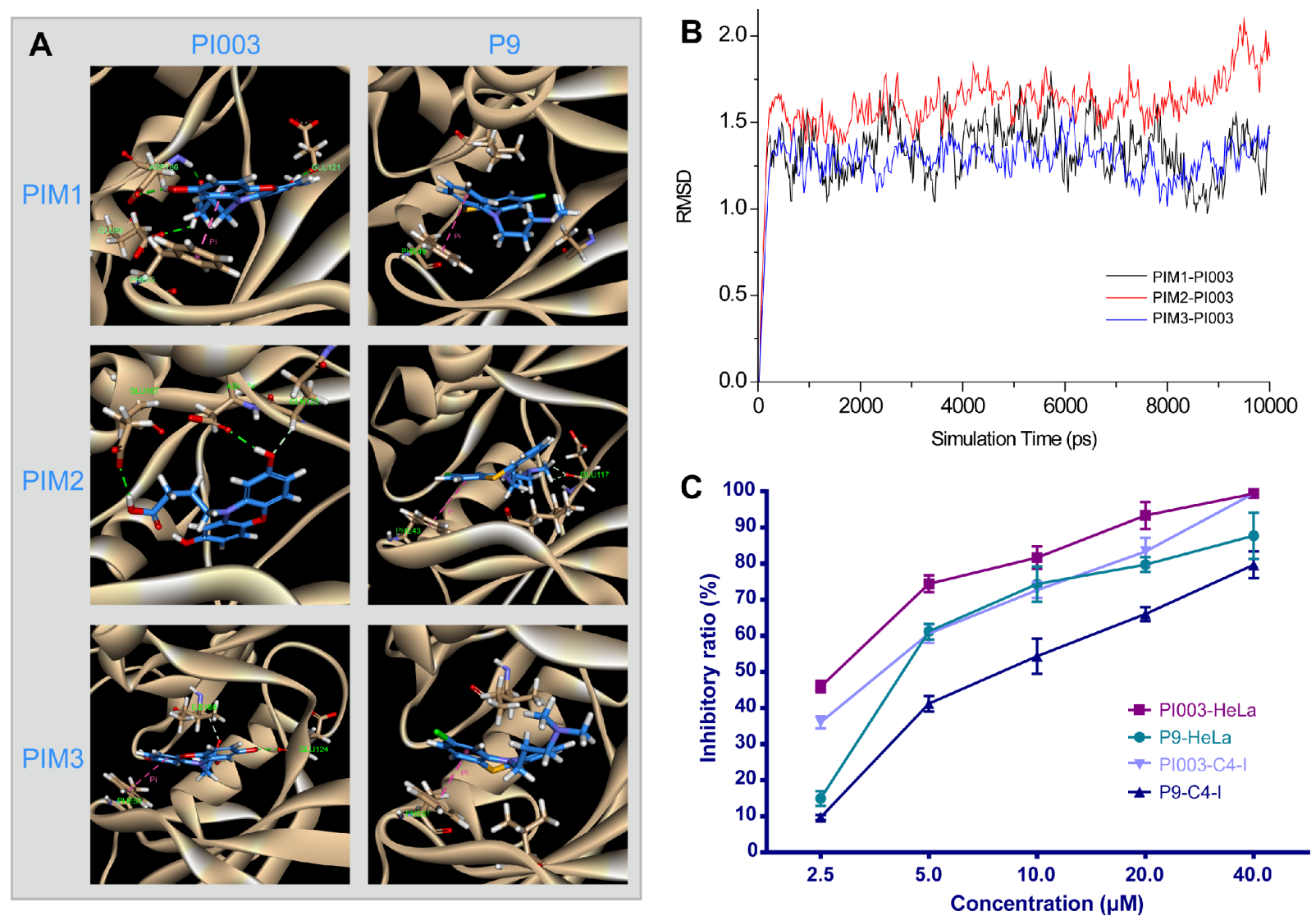

Figure 3: In silico and experimental comparisons between PI003 and P9. (A) Molecular docking of PI003 and P9. Compared with P9, PI003 has more hydrogen bonds. (B) MD simulations of PI003-PIM kinases complexes. The MD simulations were stable after $1 \mathrm{~ns}$ for the three complexes. (C) The MTT assay of PI003 and P9. The PI003 had a remarkable increase in inhibitory ratio of HeLa and C4-I cells.

\section{PI003-induced apoptotic mechanism is mainly dependent on PIM-1, but partially dependent on PIM-2 and PIM-3}

As a pan-PIM inhibitor, PI003 may target PIM1, PIM2 and PIM3. To our best knowledge, PIM1 takes the main effect of PIM kinases in cancer. So the PI003 was designed to target PIM1 mainly (Figure 6A). When treated with PI003, caspase 3, 8 and 9 level showed remarkable increasing and the level of PIM1 decreased. But when PIM-1 siRNA added, PI003 cannot increase the level of caspase 3, 8 and 9. When adding PIM-2 siRNA (Figure 6B) and PIM-3 siRNA (Figure 6C), PI003 partially increased caspase 3,8 and 9 . Thus, these results suggest that PI003-induced apoptotic mechanism is mainly dependent on PIM-1, but partially dependent on PIM-2 and PIM-3.

\section{MiR-1296/miR-1299 could affect PIM1 and STAT3 in PI003-indcued apoptosis}

We utilized a miRNA microarray analysis to identify miRNAs that were expressed in HeLa cells when treated with PI003 and 70 miRNAs were identified (Figure 7A).
Based on the differentially expressed miRNAs, a SAM analysis was used to compare the expression data of two treated samples and two normal samples (Figure 7B). A total of 35 upregulated and 2 downregulated miRNAs were identified with statistical significance in the treated samples.

Seven high conforming predicted miRNAs were selected out, Combined with apoptic proteins in the PPI of PIM kinases, the potential miRNA-PIM-gene pathways were predicted. MiR-1273a, miR-1296, miR-1299 and miR-664a-3p for PIM1 and miR-1273a, miR-1296, miR1299, miR-664a-3p, miR-1285-3p, miR-1226-3p and miR-1224-3p for PIM2 were selected. And the interacted genes which may play a role in PI003-induced apoptosis and miRNA-PIM-gene/protein pathways were selected (Figure 7C). Such as GSK3B, which has pro-apoptotic effect through phosphorylation of the anti-apoptotic protein MCL1, may control cell apoptosis in response to growth factors deprivation. To further study miRNA involvements, we found that miR-1296 mimetic and miR1299 mimetic could remarkably decrease the expressions of PIM1, suggesting that they negatively regulates PIM1 and induces apoptosis in HeLa cells (Figure 8). In addition, in PI003-treated HeLa cells, we found that 
Table 1: Binding free energies and individual energy terms of PI003 in complex with PIM-1, PIM-2 and PIM-3 (kcal/mol)

\begin{tabular}{|l|c|c|c|}
\hline Contribution & \multicolumn{1}{c}{ Pim1-PI003 } & Pim2-PI003 & $-34.00(0.54)$ \\
\hline$\Delta \mathrm{E}_{\text {int }}^{\text {ele }}$ & $-41.60(0.88)$ & $-41.83(0.99)$ & $-35.85(0.38)$ \\
\hline$\Delta \mathrm{E}_{\text {int }}^{\text {dw }}$ & $-34.71(0.37)$ & $-37.57(0.29)$ & $-5.60(0.04)$ \\
\hline$\Delta \mathrm{G}_{\text {sopol }}^{\text {nopol }}$ & $-5.54(0.02)$ & $-5.12(0.02)$ & $41.75(0.56)$ \\
\hline$\Delta \mathrm{G}_{\text {sol }}^{\text {ele }}$ & $48.18(0.65)$ & $55.91(0.85)$ & $36.15(0.61)$ \\
\hline$\Delta \mathrm{G}_{\text {sol }}$ a & $42.64(0.66)$ & $50.79(0.83)$ & $7.75(0.10)$ \\
\hline$\Delta \mathrm{G}_{\text {ele }}{ }^{\mathrm{b}}$ & $6.58(0.06)$ & $14.08(0.12)$ & $-8.2(0.09)$ \\
\hline$-\mathrm{T} \Delta \mathrm{S}$ & $-9.8(0.13)$ & $-10.2(0.18)$ & $-42.12(0.26)$ \\
\hline$\Delta \mathrm{G}_{\text {bind }}$ & $-43.69(0.32)$ & $-38.81(0.25)$ & \\
\hline
\end{tabular}

${ }^{a}$ The polar/nonpolar $\left(\Delta \mathrm{G}_{\text {sol }}^{\text {ele }}+\Delta \mathrm{G}_{\text {sol }}^{\text {nopol }}\right)$ contributions.

${ }^{b}$ The electrostatic $\left(\Delta \mathrm{E}_{\text {int }}^{\text {ele }}+\Delta \mathrm{G}_{\text {sol }}^{\text {ele }}\right)$ contributions. All energies are averaged over 200 snapshots and are given in $\mathrm{kcal} / \mathrm{mol}$. Calculation of $\Delta \mathrm{G}_{\text {bind }}$ does not explicitly consider entropy contributions. The values in parentheses represent the standard error of the mean.

Table 2: Hydrogen bonds analysis of the small-molecule inhibitor PI003 into PIM1, PIM2 and PIM3 binding sites based upon MD simulations

\begin{tabular}{|c|c|c|c|c|c|c|}
\hline Complex & Donor & Acceptor-H & Acceptor & $\%$ Occupied & Distance( $(\hat{A})$ & Angle(Degree) \\
\hline \multirow{2}{*}{ PI003-Pim1 } & $57: \mathrm{OE} 2$ & DRG:H37 & DRG:O22 & 99.6 & 2.620 & 19.44 \\
\hline & 154:OD2 & DRG:H35 & DRG:O18 & 42.2 & 2.679 & 15.11 \\
\hline PI003-Pim2 & 80:OD1 & DRG:H36 & DRG:O19 & 74.3 & 2.660 & 19.31 \\
\hline \multirow{3}{*}{ PI003-Pim3 } & 150:OD2 & DRG:H36 & DRG:O19 & 97.3 & 2.682 & 16.71 \\
\hline & 51:OE2 & DRG:H37 & DRG:O22 & 95.2 & 2.626 & 27.04 \\
\hline & DRG:O21 & $150: \mathrm{H}$ & $150: \mathrm{N}$ & 25.8 & 2.836 & 27.93 \\
\hline
\end{tabular}

miR-1296 and miR-1299 mimetics could remarkably decrease the expression of p-STAT3, which has been predicted to interact with PIM1 Figure 8. Thus, these results indicate that the two miRNAs can target PIM1mediated apoptotic pathway in HeLa cells.

\section{Anti-tumor activity and apoptosis-inducing effect of PI003 in vivo}

Based on the anti-proliferative efficacy of PI003 on HeLa cells in vivo, we proceeded to assess its efficacy on inhibiting tumor growth in an orthotopic xenograft mouse model of cervical cancer. In this experiment, we used three different doses of PI003. Compared with the control group, median and high doses of PI003 can induce the significant body weight loss in nude mice in a dose dependent manner $(P<0.001)$, while the toxicity of low dose PI003 was not obvious (Figure 9A). At the end of the experiment, the tumor weights decreased remarkably in median and high dose groups (median dose group, $P<0.05$; high dose group, $P<0.01)$. For more toxicity study, spleen weights were affected by different doses of PI003 $(P<0.05)$, the decrease of liver and kidney weights were not obvious. We obtained identical results by directly measuring the tumor volumes. In the three PI003 groups, the tumor volumes were much smaller than the control group in all three doses groups (Figure 9B). In according to the balance between anti-tumor efficacy and toxicity, the median dose was used as the optimum dose for treatment of tumor growth. In addition, PI003 administration resulted in a statistically significant increase in number of apoptotic bodies in the tumor as visualized by TUNEL assay, suggesting that PI003-induced tumor cell proliferation inhibition by apoptosis (Figure 9C).

\section{DISCUSSION}

PIM kinase inhibitors were frequently reported as a new class of cancer therapeutics recently. PIM kinase are regulated primarily by transcription and stability 
A
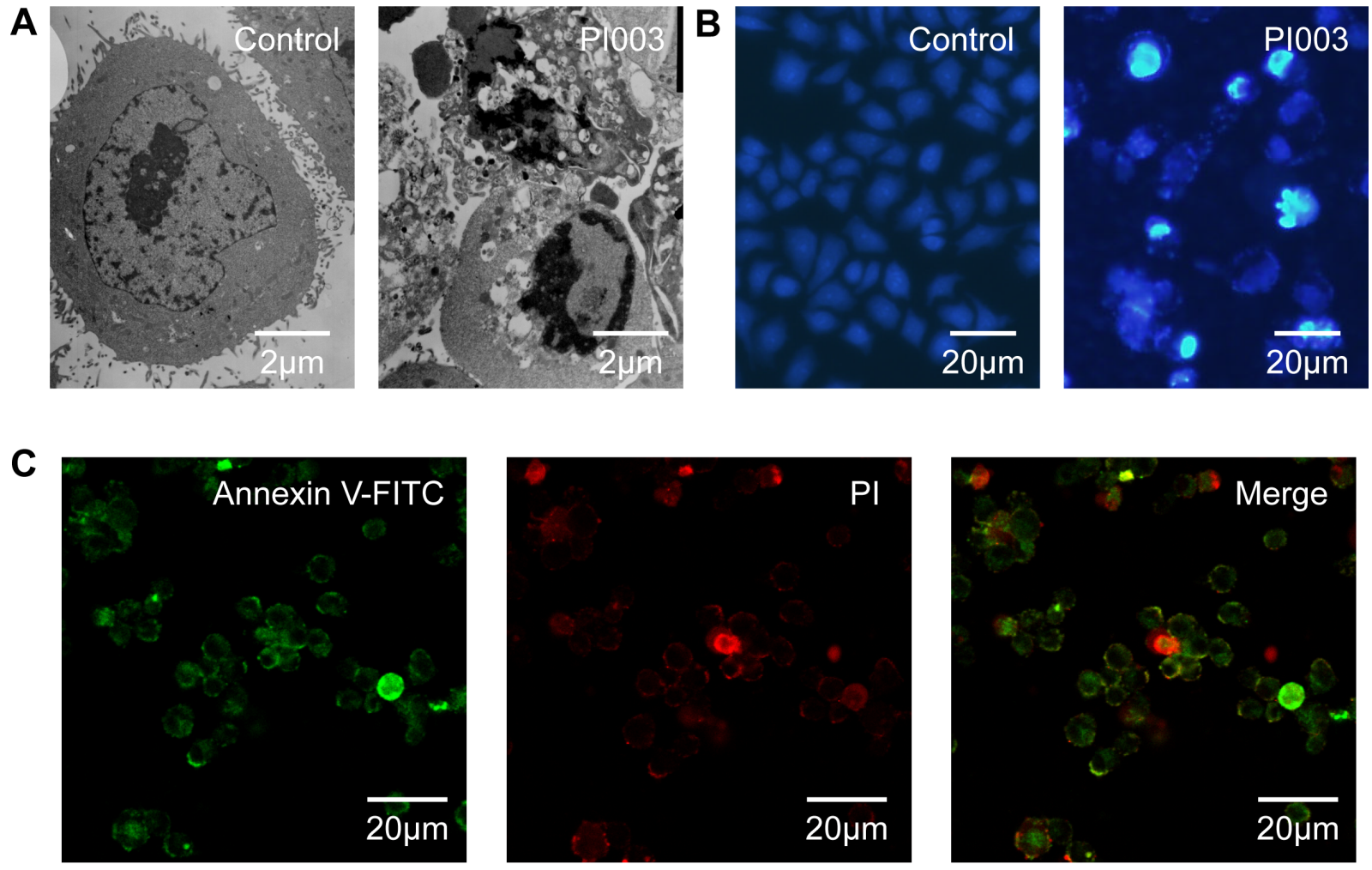

D
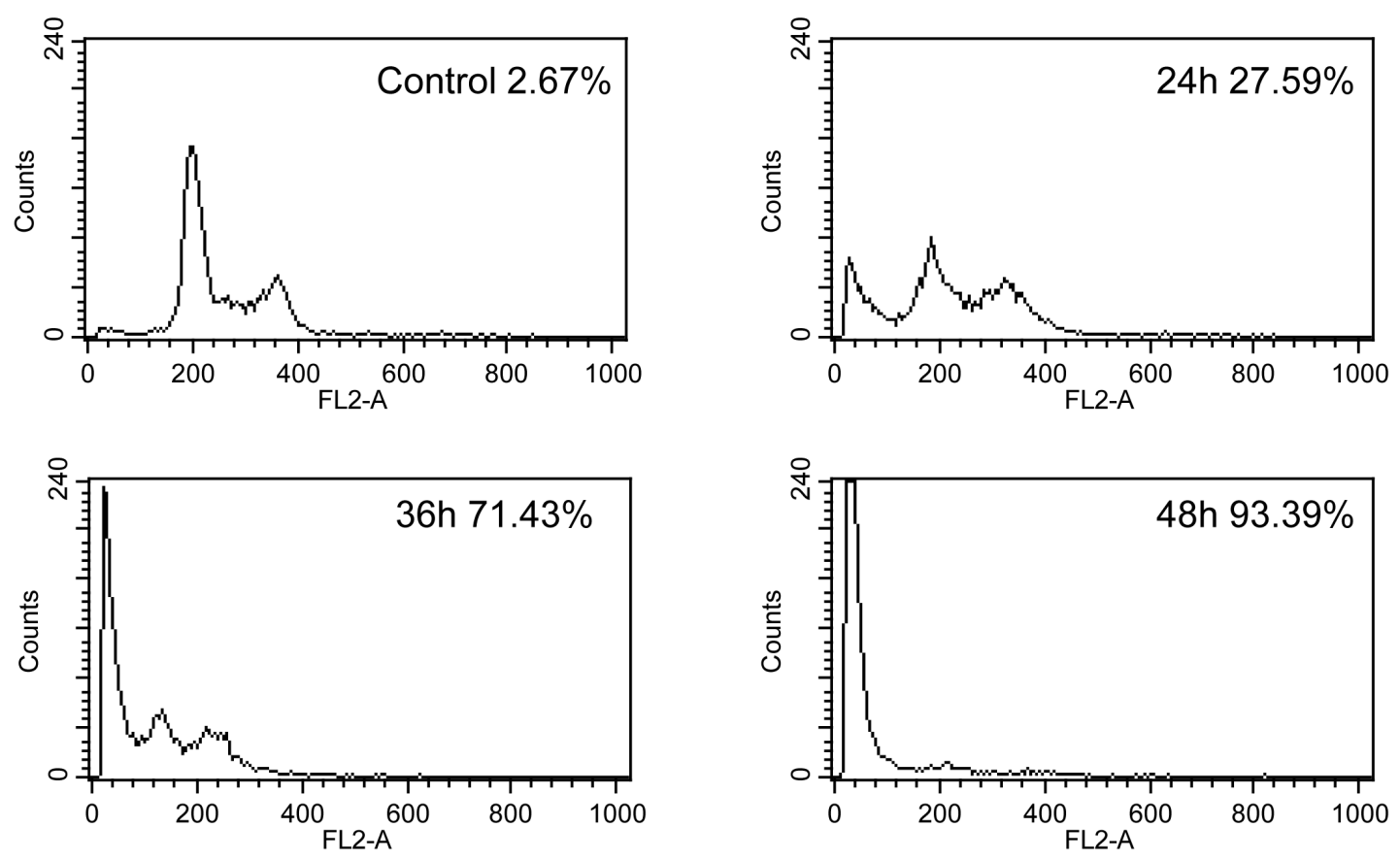

Figure 4: PI003 induces apoptosis in HeLa cells. (A) The cellular morphology was observed without or with PI003 under the inverted microscopy. (B), (C) Apoptosis was determined by the analyses of Hoechst 33342 staining and Annexin staining. (D) The population of SubG1 cells was measured by flow cytometry after collection.

through pathways that are controlled by JAK/STAT, transcription factors. They are interesting targets for new drug development for the overexpressing in many cancers and involving in cancer-specific pathways, such as cell survival, cell cycle progression and cell migration.
It has being reported that increased expression of PIM contributed to increasing the susceptibility of leukemia, lymphoma, hepatocarcinoma and prostatic carcinoma. PIM kinase inhibitors are emerging as a new class of cancer therapeutics, and the prevalence of increased PIM 


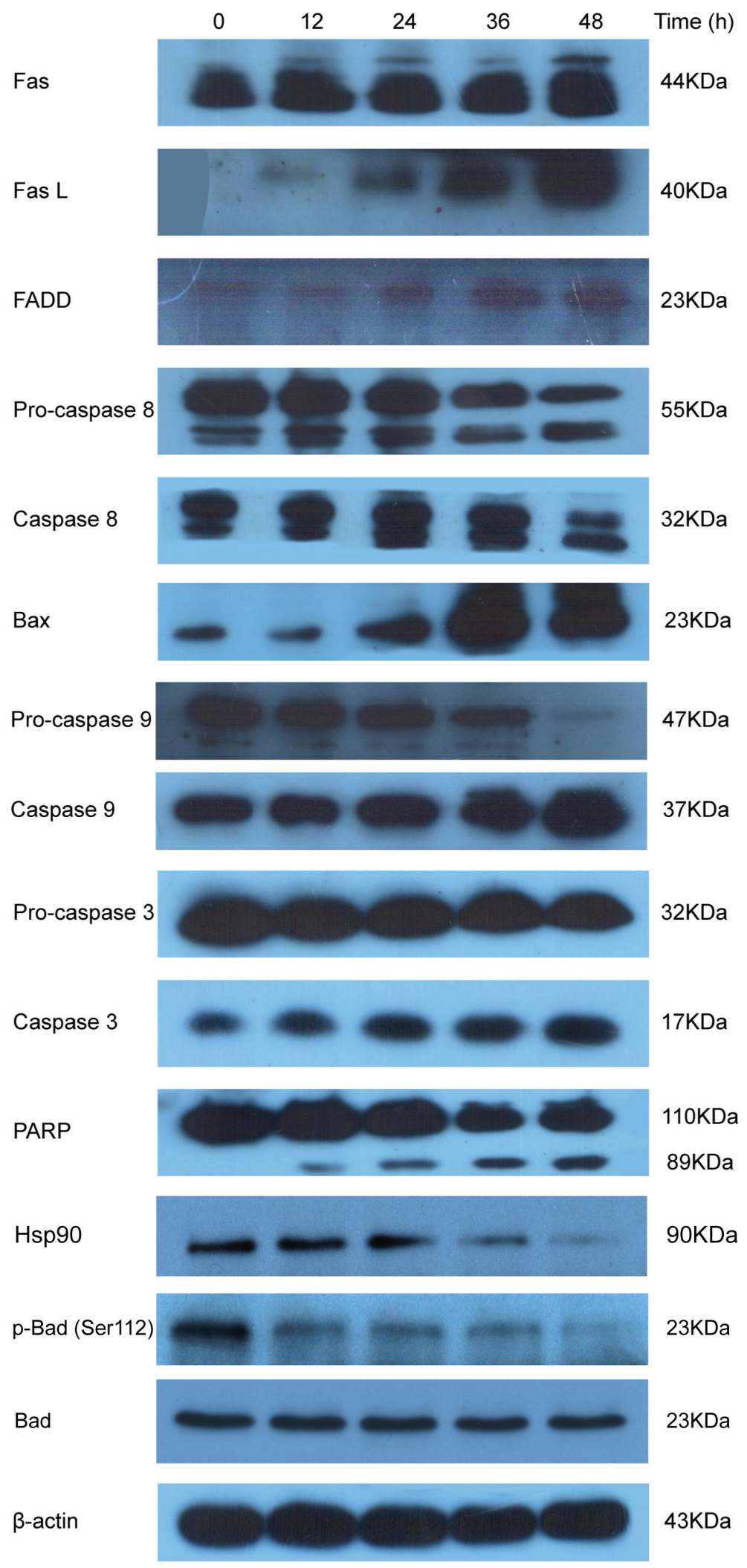

Figure 5: PI003 induces HeLa cell apoptosis via the death-receptor and mitochondrial pathways. 


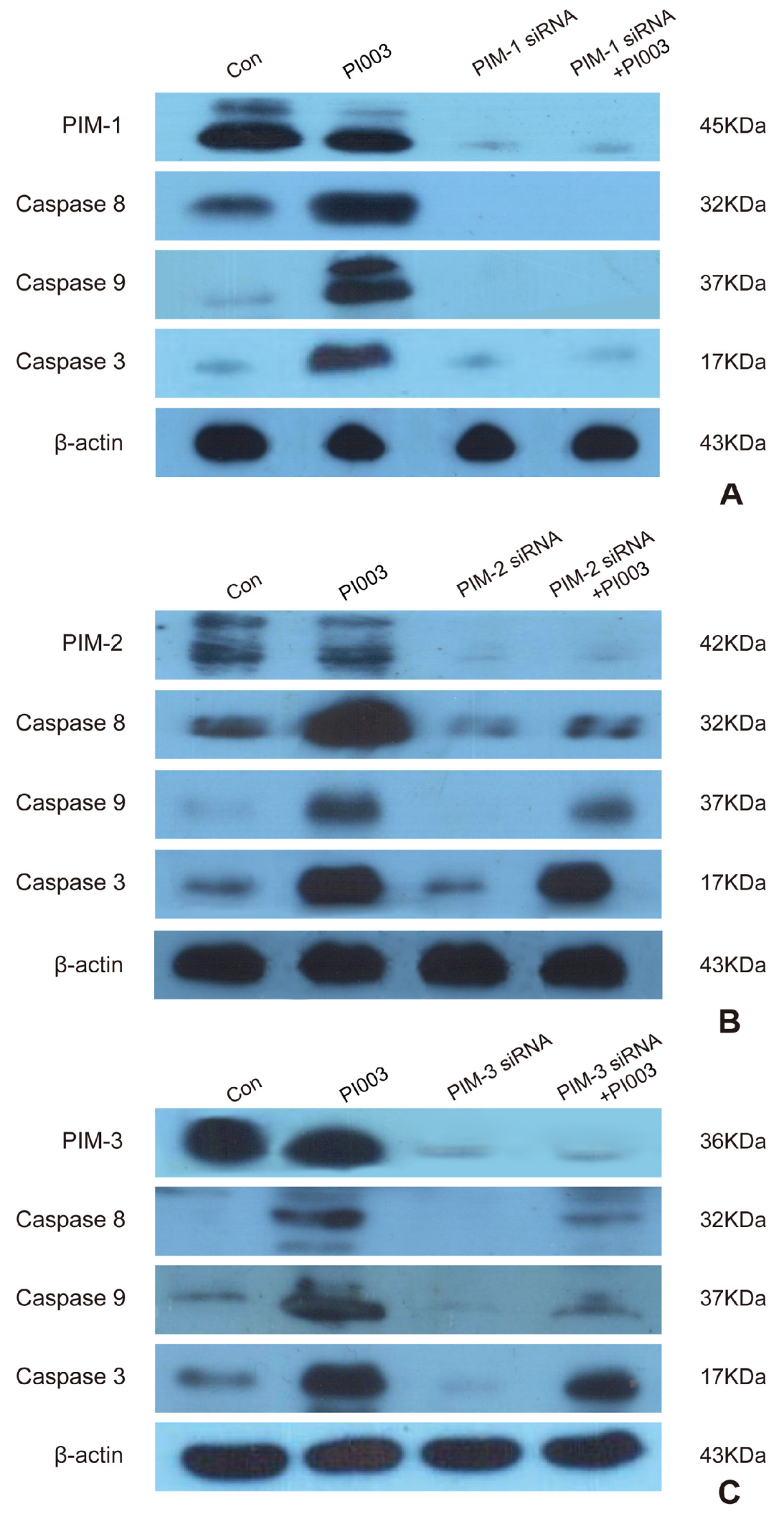

Figure 6: PI003 induces apoptosis by targeting PIM1, PIM2 and PIM3. (A) PI003-induced apoptosis is mainly dependent on PIM1; (B) PI003-induced apoptosis is partially dependent on PIM2; (C) PI003-induced apoptosis is partially dependent on PIM3. 

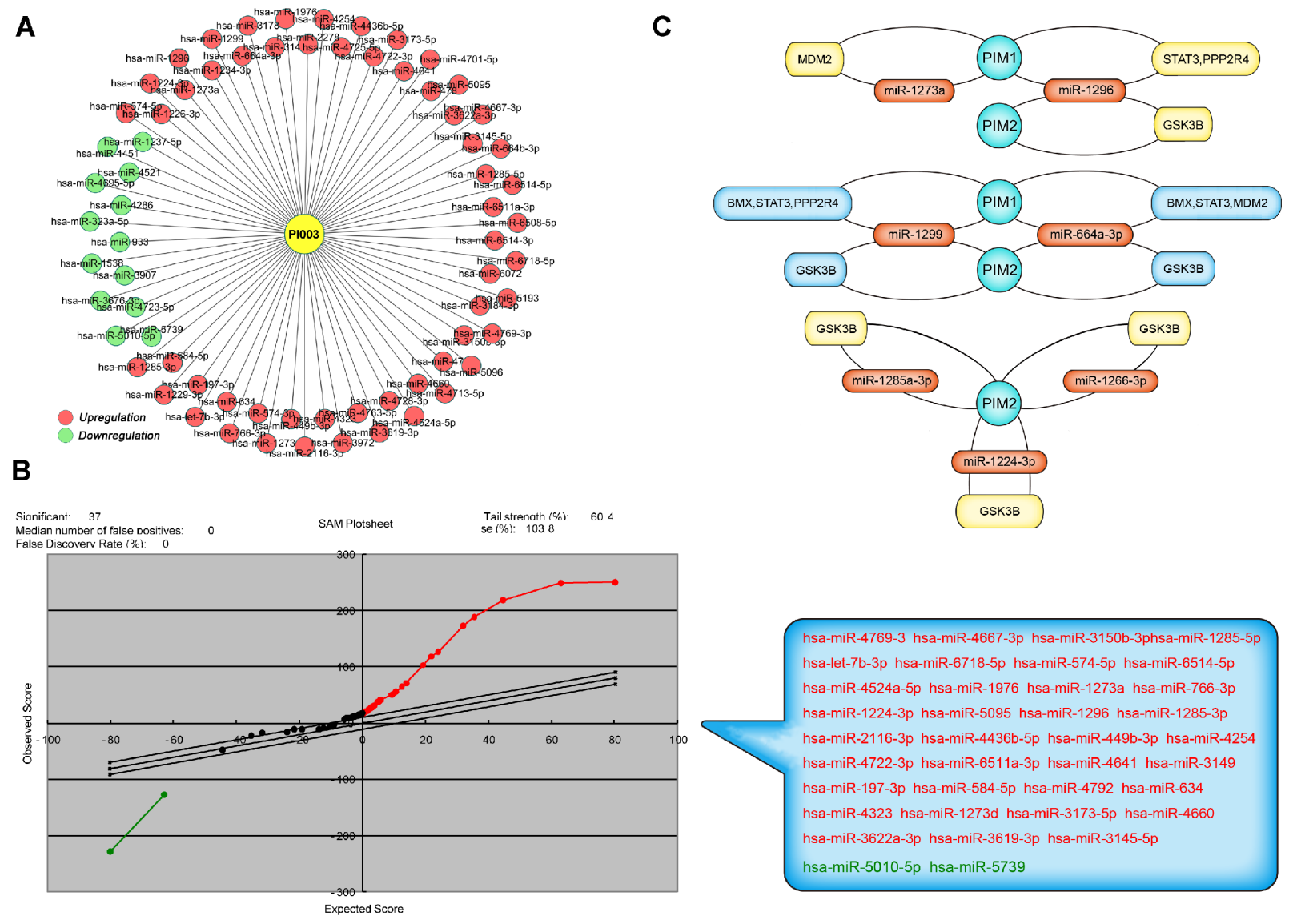

Figure 7: Microarray-based analysis of PI003-induced apoptotic mechanisms involved in microRNAs. (A) MiRNA microarray analysis of PI003-induced HeLa cell apoptosis. (B) The SAM analysis was used to compare the expression data, 37 miRNAs were identified with statistical significance in the treated samples. (C) The key-interacted genes in PIM-miRNA-gene/protein apoptotic pathways.

kinases expression across different cancer types suggests that PIM inhibitors may be a treatment modality for a variety of cancer [14]. There no research of PIM inhibitors for cervical cancer reported to date. It is a challenge to discover inhibitors of PIM kinases in cervical cancer therapeutics. When PIM1 was inhibited, PIM2 expression increased across different cancer types generally, and Pim3 was reported to be aberrantly expressed in colon cancer [15]. Taken together, designing pan-PIM inhibitor was necessary for Pim-related cancer therapeutics.

In our study, we developed the Naïve Bayesian model, which was well-suited to integrate these highthroughput data such as smallest shared biological processes (SSBP), gene co-expression profiles, domaindomain interaction (DDI) and cross-species interolog mapping for predicting protein functional connections; thereby, constructing the PIM kinase network. It is wellknown that Naïve Bayesian model is used for constructing the global PPI network, suggesting that this mathematics model is advantageous for elucidating the global PPI network in model organisms. Compared to previous studies for the discovery of pan-PIM compounds, the mechanism of PIM inhibitors induced apoptosis was further identified. We identified more novel interacted proteins with PIM kinases (PIM1, PIM2 and PIM3) and further shed light on more molecular mechanisms of PIM kinases in cancer. The other notable result is the binding mode of PI003. Generally, the inhibitors bind to the active site of AMP (Supplementary Figure S1), while in PIM1-PI003 complex, PI003 had Pi interaction with PHE49 and hydrogen bond interaction with GLU89. This binding mode can be used to aid in the design of specific inhibitors of PIM inhibitors.

In this study, FDA-approved small-molecule compounds were screened for candidate drugs and PI003 was modified from the best one, P9, and more hydrogen bonds were formatted to increase the stability. Compared with other pan-PIM inhibitors, such as 7-(4H-1,2,4-Triazol-3-yl)benzo[c] [2, 6] naphthyridines and pyrazolo[1,5-a]pyrimidine-based Pim inhibitors PI003, modified from a FDA-approved drug, had more druggability $[16,17]$. A series of experimental data have demonstrated that PI003-induced apoptosis was the death-receptor and mitochondrial pathways in HeLa cells. ETP-45299, which was previously described as a PIM1 inhibitor in various human tumor cells was not tested in 


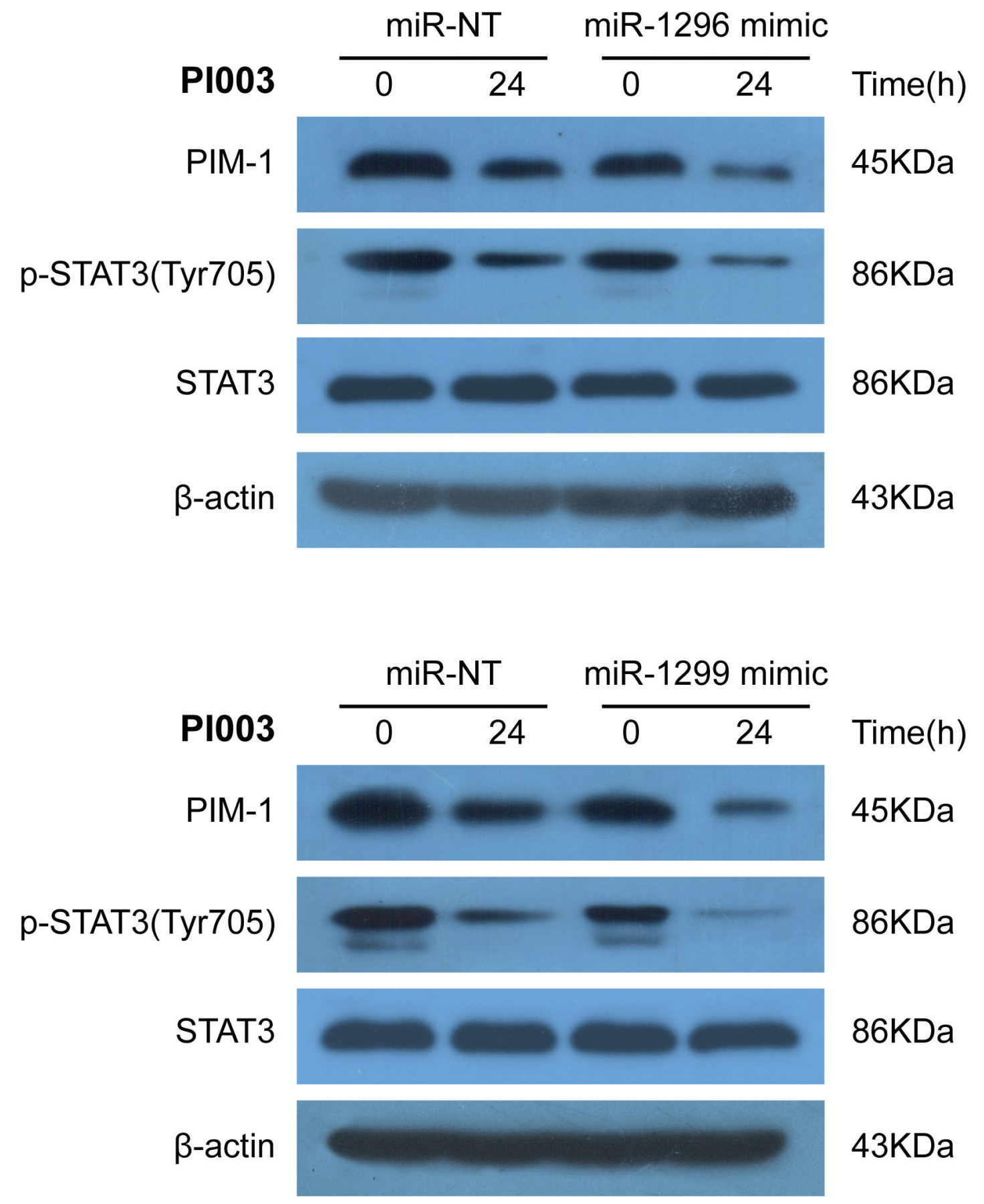

Figure 8: Identification of miR-1296- and miR-1299-regulated mechanisms in PI003-induced apoptosis. MiR-1296 mimetic and miR-1299 mimetic could remarkably decrease the expressions of PIM1 and p-STAT3 in PI003-treated HeLa cells.

HeLa cells. While the PI003 was firstly desired pan-PIM inhibitor in HeLa cells [13]. The evidence was clearer than single PIM kinase inhibitory activity analysis of other PIM inhibitors, such as benzylidene-1,3-thiazolidine2,4-diones [18]. As the results of western blot analysis, apoptosis via both death-receptor and mitochondrial pathways was identified. Compared with examined the phosphorylation of BAD, like the CX-6258 [19] and LGB321 [20], we clearly explained that the apoptotic mechanisms of PI003. Of note, Pan-PIM inhibitors are valuable for cancer therapeutics. PIM1, PIM2 and PIM3 had different sensitivity of PI003, this design combined the pan-PIM inhibition and low cytotoxicity. Compared with SGI-1776 [21] and AZD1208 [22], in PI003-treated HeLa cells, some new miRNA-regulated mechanisms were also reported. The high differentially expressed miRNAs from microarray with predicted effect of target PIM kinases may play a key role in regulating PIM 

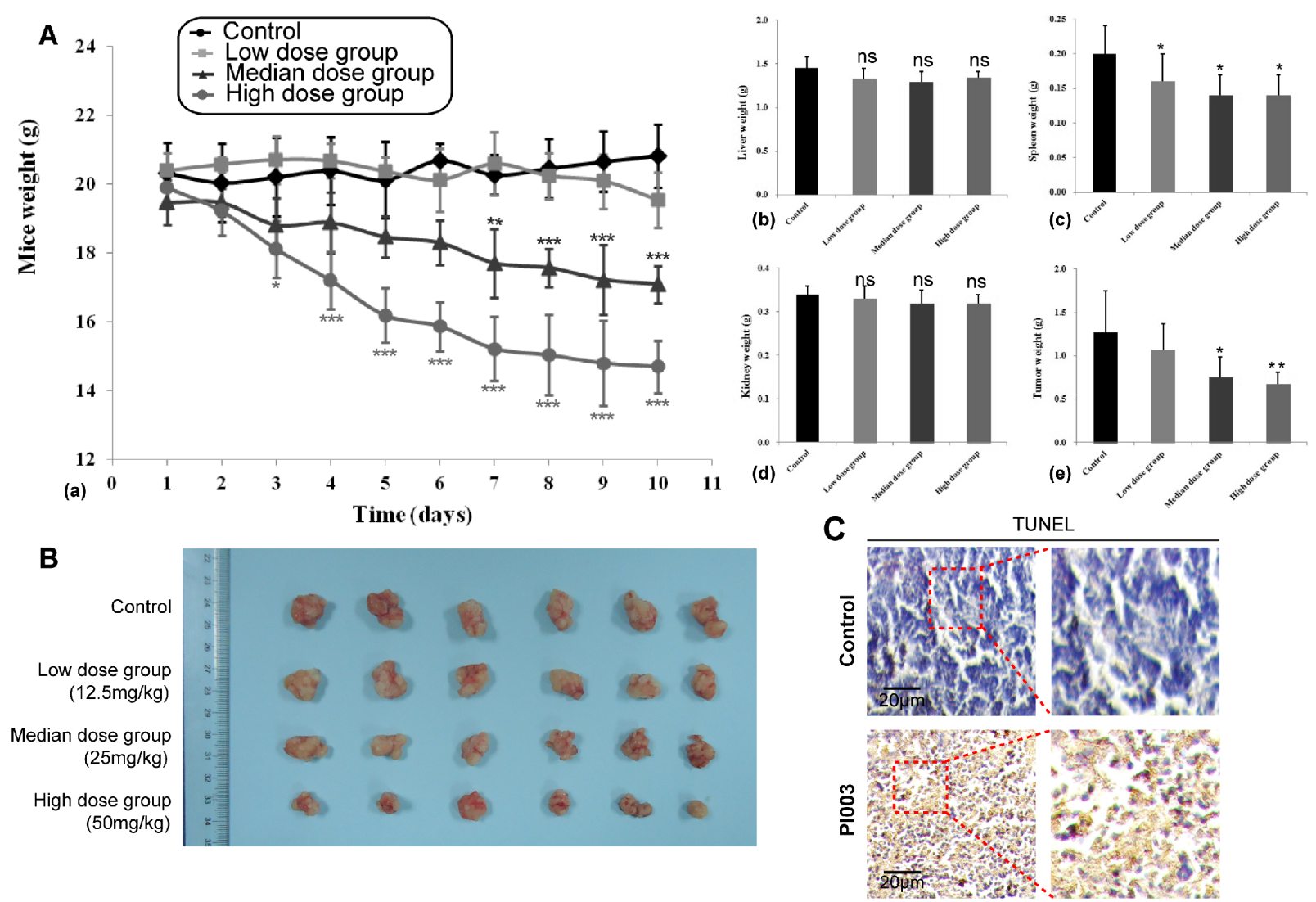

Figure 9: Anti-tumor activities of PI003 in in vivo mouse models. (A) Anti-tumor activities of PI003 and its toxicity. The treatments began on day 1 after grouping (day 0), including vehicle, low dose of PI003 $12.5 \mathrm{mg} / \mathrm{kg}$ once a day, median dose of PI003 25 $\mathrm{mg} / \mathrm{kg}$ once a day and high dose of PI003 $50 \mathrm{mg} / \mathrm{kg}$ once a day for 10 days; (B) The inhibitory rate of tumor. Representative tumors from mice after vehicle and PI003 treatment; (C) TUNEL immunohistochemistry in representative tumor section of a control mouse and a mouse of the median dose group.

signaling pathway. The predicted miRNA-PIM-gene pathways, contended the validated interacted proteins of PIM kinases. This results were carried out for the first time and may be experimentally verified in miRNA level. For instance, PIM2 target miRNA, miR-1226-3p, which was verified by NGS (http://mirtarbase.mbc.nctu.edu.tw/), was in one of our predicted miRNA-PIM-gene pathways. For instance, we predicted miR-1296/miR-1299-PIM1-STAT3 pathway, and thus validated the two miRNA mimetic to inhibit the expressions of PIM1 and p-STAT3 in PI003induced apoptosis of the HeLa cells. This work may shed light on the further research on the combinations of panPIM kinases and miRNA drugs in future cancer therapy. Moreover, in our study, we also found that PI003 bear the good anti-tumor activities, and also induced apoptosis in vivo. It may provide a board perspective for utilizing PI003 as a potential anti-tumor drug targeting PIMs in the near future.

In conclusion, these findings demonstrate that PI003, as a novel synthesized pan-PIM inhibitor, induces the death-receptor and mitochondrial apoptosis involved in some microRNA regulation, and also possessed remarkable anti-tumor activity and apoptosis-inducing effect in vivo. Thus, these findings would provide us a new clue on discovering more potential small-molecule pan-PIM inhibitors in future cervical cancer therapy.

\section{MATERIALS AND METHODS}

\section{Network construction}

To build the protein-protein interaction (PPI) network of PIM kinases, we collected a diverse of datasets from online databases. The globe network data were from Human Protein Reference Database (HPRD) [23], Biomolecular Interaction Network Database (BIND) [24], the Database of Interacting Proteins [25], Munich Information center for Protein Sequences [26], IntAct [27] and PrePPI [28]. Then, we applied a Naïve Bayesian model to integrate diverse data and thus making the final interaction predictions in a systematic way [29]. Following the Naïve Bayesian theorem, we compute the posterior odds given $\mathrm{n}$ evidence as follows:

$$
O_{\text {posterior }}=\frac{P\left(\text { positive } \mid E_{1, \ldots,} E_{n}\right)}{\mathrm{P}\left(\text { negative } \mid E_{1, \ldots,} E_{n}\right)}
$$


Where positive means that two proteins are functional related while negative means not.

$$
\begin{aligned}
& \text { We define } \operatorname{LR}_{\left(E_{1}, \ldots, E_{n}\right)}=\frac{P\left(E_{1, \ldots,}, E_{n} \mid \text { positive }\right)}{P\left(E_{1, \ldots}, E_{n} \mid \text { negative }\right)} \text { then } \\
& O_{\text {posterior }}=O_{\text {prior }} \times \mathrm{LR} \text {. }
\end{aligned}
$$

As Naive Bayesian model supposes that each evidence is conditional independent, we can simplify LR as

$$
\operatorname{LR}_{\left(E_{1}, \ldots, E_{n}\right)}=\prod_{i=1}^{n} L R(E i)
$$

A likelihood ratio (LR) corresponding to a specific biological evidence (Ei) was used to measure the predictive power or confidence degree, and calculated as the ratio of the true positive rate (TPR) to the falsepositive rate $(\mathrm{FPR})$, where $\mathrm{TPR}=|\mathrm{EiCPRS}| /|\mathrm{PRS}|$ and $\mathrm{FPR}=\mid$ EicNRS $|/| \mathrm{NRS} \mid$. In theory, LR (Ei) $>1$ indicates that the biological evidence Ei is capable of identifying the true positives from a test. The LR was calculated from four evidences: (A) Interology mapping of 6 model organisms. (B) Gene co-expression data calculated from high quality large-scale microarray datasets. (C) Domaindomain interaction data from PrePPI. (D) Smallest shared biological processes that quantify protein biological process similarity. Based on these evidences, best predicted proteins are chosen by preset scores. And a ROC curve allows us to explore the relationship between the sensitivity and specificity of a binary classifier system for a variety of different cut points [30].

\section{Molecular docking}

FDA-approved small molecule compounds from the latest version of DrugBank (http://www.drugbank. $\mathrm{ca} /$ ) were downloaded to construct the screening library for PIM kinases. In addition, we used Accelrys Discovery Studio version 3.5 (Accelrys Inc., USA) with CHARMm force-field parameters to dock pre-generated conformations of drugs into PIM1, PIM2 and PIM3 for virtually screening potential candidate inhibitors. We performed flexible-ligand docking to a rigid receptor with grid-based scoring, in which drugs were allowed to be flexible and structurally rearranged in response to PIM1, PIM2 and PIM3. The P1-P20 were selected based on their scores and structures.

\section{Cell culture and the MTT assay}

The HeLa cells and C4-I cells were cultured in Dulbecco's modified Eagle's medium (DMEM) with 10\% (v/v) heat-inactivated fetal bovine serum and incubated in a humidified incubator with $5 \% \mathrm{CO}_{2}$. HeLa cells and C4-I cells were transferred to 96-well plates at a density of approximately $5.0 \times 10^{4}$ cells $/ \mathrm{mL}$. Cytotoxicity induced by the test compounds were measured using the MTT assay as follows: The HeLa cells and C4-I cells were cultured in DMEM in the presence of $30 \mu \mathrm{M}$ test compound for $24 \mathrm{~h}$ and then 10ul of MTT ( $5 \mathrm{mg} / \mathrm{ml})$ was added to cells in each well. After $4 \mathrm{~h}$ of culture, the medium was removed, and the blue formazan crystals that had formed were dissolved in dimethyl sulfoxide. The absorbency of formazan generated from MTT was measured at $570 \mathrm{~nm}$ (BioRad Model 680, Bio-Rad, Hercules, CA, USA) [31, 32]. The inhibitory ratio $(\%)=\left(\mathrm{OD}_{490 \text {, control }}-\mathrm{OD}_{490, \text { sample }}\right) /$ $\left(\mathrm{OD}_{490, \text { control }}-\mathrm{OD}_{490, \text { blank }}\right) * 100$.

\section{Chemical synthesis}

All reactions requiring anhydrous conditions were performed under an Ar or N2 atmosphere. Chemicals and solvents were either A.R. grade or purified by standard techniques. Thin layer chromatography (TLC): silica gel plates GF254; compounds were visualized by irradiation with UV light and/or by treatment with a solution of phosphomolybdic acid (20\% wt. in ethanol) followed by heating. Column chromatography was performed by using silica gel with eluent given in parentheses. 1H NMR analysis was performed using $\mathrm{CDCl} 3$ or DMSO-d6 as a solvent at room temperature. The chemical shifts are expressed in relative to TMS $(=0 \mathrm{ppm})$ and the coupling constants $\mathrm{J}$ in Hz. The purity of compound screened in biological assays was determined to be $\geqq 97 \%$ by HPLC analysis with a photodiode array detector, An atlantis C18 $(150 \mathrm{~mm} \times 4.6 \mathrm{~mm}$, i.d. $5 \mu \mathrm{m})$ (Waters, Milford, Mass, USA) was used with a gradient elution of methanol and HPLC-grade water as mobile phase at a flow rate of $1 \mathrm{ml} /$ $\min [33,34]$.

\section{Molecular dynamics (MD) simulations}

MD simulations were performed with GROMACS (version 4.5.5) software package [35] to monitor the binding states between PIM kinases and PI003. The topology parameters of ligands were built by the Dundee PRODRG server [36]. The topology of PIM kinases was edited by Amber force field 99SB and the small molecule was edited by Amber general force field. The complexes were immersed in a cubic box of simple point charge (SPC) water molecules. Eight and eleven sodium counterions were added by replacing water molecules to ensure the overall charge neutrality of the receptors simulated system, respectively. In this MD process, $1 \mathrm{~ns}$ simulations with a time step of $10 \mathrm{~ns}$ were performed, and the resulting trajectory files were viewed and analyzed using VMD software [37].

\section{Apoptosis detection}

The ultrastructure of cell apoptosis was observed under an electron microscope (Hitachi 7000, Japan). Hoechst 33342 staining and Annexin V-FITC staining were performed to detect apoptosis. For Hoechst 33342 staining, A549 cells were washed with PBS and stained with Hoechst $33342(1 \mu \mathrm{g} / \mathrm{ml}$ in PBS) at room 
temperature for $20 \mathrm{~min}$, the fluorescence was observed by a fluorescence inverted microscopy. For Annexin V-FITC staining, the treated cells were collected, washed and then stained with Annexin V-FITC or PI at room temperature for $15 \mathrm{~min}$. The percentage of apoptotic cells were analyzed by flow cytometry (Becton Dickinson, Franklin Lakes, NJ) [38-40].

\section{Western blot analysis}

Cellular proteins were extracted using RIPA buffer (SolarBio, 50 mM Tris/HCl, pH 7.4, 150 mM NaCl 1\% $(\mathrm{v} / \mathrm{v})$ NP-40, 0.1\% (w/v) SDS) containing 1\% (v/v) PMSF (SolarBio), 0.3\% (v/v) protease inhibitor (Sigma) and $0.1 \%(\mathrm{v} / \mathrm{v})$ phosphorylated proteinase inhibitor (Sigma). Lysates were centrifuged at $12,000 \mathrm{rpm}$ at $4^{\circ} \mathrm{C}$ for 15 min and the supernatant was collected for total protein. A BCA protein assay kit (Pierce) was used to determine the protein concentration. Equal amounts of protein $(15 \mu \mathrm{g})$ was separated on an SDS-PAGE gel (10\% (v/v) polyacrylamide) and transferred onto a PVDF membrane. Nonspecific binding was blocked using 8\% (w/v) milk in TBS-T for $2 \mathrm{hr}$ at room temperature. The membranes were then incubated with primary antibodies overnight at $4^{\circ} \mathrm{C}$. After several washes with TBS-T, the membranes were incubated in HRP-conjugated goat anti-rabbit and anti-mouse IgG or HRP-conjugated mouse anti-goat IgG (Abmart, all at a 1:5000 dilution) for $2 \mathrm{hr}$ at room temperature and then washed. The target proteins were visualized using enhanced chemiluminescence (Millipore) according to the manufacturer's recommendations, and quantified using density analysis normalized against GAPDH to the manufacturer's recommendations, and expressed as the fold-change compared to control.

\section{SiRNA and miRNA transfection}

Small interfering RNAs (siRNAs) against human PIM-1, PIM-2 and PIM-3, and control siRNA were purchased from Invitrogen (Carlsbad, CA). MiR-1296 mimetic and miR-1299 mimetic were purchased from Sigma. The HeLa cells were transfected with siRNAs at $33 \mathrm{nM}$ final concentration using Lipofectamine 2000 (Invitrogen) according to the manufacturer's instructions. For the miRNA transfection, HeLa cells were transfected with miR-1296 mimetic and miR-1299 mimetic at 100 nM final concentration using Lipofectamine RNAiMAX reagent (Invitrogen) according to the manufacturer's instructions. The transfected cells were used for subsequent experiments $24 \mathrm{~h}$ later.

\section{MicroRNA microarray and SAM analysis}

Human OneArray ${ }^{\circledR}$ microarrays were pre-heated at $60^{\circ} \mathrm{C}$ for $10 \mathrm{~min}$ in hybridization oven. Microarray slides were placed inside a falcon tube containing 100\% ethanol, incubated for approximately $15 \mathrm{sec}$, shaken for $20 \mathrm{sec}$, and thoroughly rinsed with deionized water to remove any residual ethanol. Next, the microarray slides were fully submerged in an abundant amount of pre-hybridization solution (5X SSPE, $0.1 \% \mathrm{SDS}$, and 1\% BSA) for $1 \mathrm{hr}$ at $42^{\circ} \mathrm{C}$. After $1 \mathrm{hr}$, slides were transferred to roomtemperature distilled water and washed gently for $2 \mathrm{~min}$. Slides were spun dry for $2 \mathrm{~min}$ and stored in a dry and dark place until hybridization.

$10 \mu \mathrm{g}$ of cRNA was fragmented by using RNA Fragmentation Reagent kit (AM\#8740, Ambion Inc., Austin, Texas, USA), and then denatured in a PCR machine at $95^{\circ} \mathrm{C}$ for 5 minutes and held at $60^{\circ} \mathrm{C}$. Fragmented cRNA was hybridized on the rice OneArray ${ }^{\circledR}$ (Phalanx Biotech Group, Taiwan) at $50^{\circ} \mathrm{C}$ for $14-16$ hrs. After hybridization, the microarrays were washed sequentially in $2 \mathrm{X}$ SSC containing $0.2 \% \mathrm{SDS}$ solution for $5 \mathrm{~min}$ at $42^{\circ} \mathrm{C}, 2 \mathrm{X} \mathrm{SSC}$ for $5 \mathrm{~min}$ at $42^{\circ} \mathrm{C}$, and $2 \mathrm{X} \mathrm{SSC}$ for $5 \mathrm{~min}$ at room temperature. Finally, the microarrays were spun dry with a centrifuge for at least one minute and stored dry in the dark until ready for scanning [41].

The significant analysis of microarray (SAM) method was used to perform the unsupervised calculation. The statistical technique is based on a $t$-test for finding significant miRNAs in a set of microarray experiments and was proposed [42]. A hierarchical clustering of the differentially expressed miRNAs was performed with Cluster 3.0 (http://bonsai.hgc.jp/ mdehoon/software/ cluster/software) version using the average linkage algorithm. The top scoring pair (TSP) algorithm was used to perform the supervised calculation [43]. The basic principle of the k-TSP is to identify miRNA pairs that are oppositely expressed (one upregulated and one downregulated) in two classes. All numerical analyses that are presented were performed using Matlab 7.0 (MathWorks Company, Natick, MA, USA).

Target miRNAs that bound to PIM kinases were predicted using miRWalk, a database on predicted and validated microRNA targets, so did the predicted targets of miRNAs [44]. Data from 9 databases (DIANA-mT [45], miRanda [46], PICTAR5 [47], miRDB [48], PITA [49], miRWalk [50], RNA22 [51], RNAhybrid [52] and TargetScan [53]) was used for identifying the high conforming predicted miRNAs. The potential miRNAPIM-gene/protein pathways were predicted by the combining of the predicted targets of conforming miRNAs and the apoptotic proteins in PPI network of PIM kinases.

\section{Mouse experiments and tumor xenograft model}

The Institutional Animal Care and Treatment Committee of No.150 Central Hospital of PLA approved all studies herein. 24 healthy female nude mice (BALB/c, 6-8 weeks of age, non-fertile and 18-20 g each) were injected subcutaneously with HeLa cells $\left(1 \times 10^{7}\right.$ cells/ mouse). When the tumors reached $100 \mathrm{~mm}^{3}$ in volume (calculated as $\mathrm{V}=\mathrm{L} \times \mathrm{W}^{2} / 2$ ). The mice were divided into four groups. Three groups were treated with different dose 
groups of PI003 once a day by intraperitoneal injection for 10 days (low dose group, $12.5 \mathrm{mg} / \mathrm{kg}$; median dose group, $25 \mathrm{mg} / \mathrm{kg}$; high dose group, $50 \mathrm{mg} / \mathrm{kg}$ ), whereas the control group was treated with vehicle control $(5 \% \mathrm{CMC}$ $\mathrm{Na}$ ). Body weight was determined every day until the end of the study. At the end of the treatment, all mice were sacrificed. Tumor tissue, spleen, liver and kidney were harvested, weighed, and photographed.

\section{TUNEL assay}

For TUNEL assay, sections were permeabilized with $0.1 \%$ Trition $\mathrm{X}-100$ plus $0.1 \%$ sodium citrate and then incubated with $50 \mathrm{ml}$ TUNEL reaction mixture (Roche) at $37^{\circ} \mathrm{C}$ for $60 \mathrm{~min}$. After rinsing with PBS three times, $50 \mathrm{ml}$ converter-POD was added and the tissue cells were incubated in a humidified chamber for $30 \mathrm{~min}$ at $37^{\circ} \mathrm{C}$. DAB substrate was then added, followed by counterstaining with hematoxylin. The assay included negative controls (without terminal transferase).

\section{Statistical analysis}

All the presented data and results were confirmed in at least three independent experiments. The data are expressed as means \pm SD. Statistical comparisons were made by Student's $t$-test. $p<0.05$ was considered statistically significant.

\section{ACKNOWLEDGMENTS}

We thank Dr. Hao Peng (Beijing University of Chinese Medicine) for his critical review on this manuscript.

\section{CONFLICTS OF INTEREST}

We declare that we have no conflicts of interest.

\section{AUTHOR CONTRIBUTIONS}

Z.L., W.H., and C.G. designed the experiments and wrote the manuscript. X.H., J.G., and J.L. performed the experiments and analyzed the data. X.H. All the authors reviewed the manuscript.

\section{ABBREVIATIONS}

PIM - serine/threonine-protein kinase, LR - a likelihood ratio, TPR - the true positive rate, FPR - the false-positive rate, FDA - Food and Drug Administration, DMEM - Dulbecco's modified Eagle's medium, MTT - 3-(4,5-dimethylthiazol2-yl)-2, 5-diphenyltetrazolium bromide, MD - molecular dynamics, PMSF - Phenylmethanesulfonyl fluoride, PVDF polyvinylidene difluoride, SDS-PAGE - polyacrylamide gelelectrophoresis, SAM - significant analysis of microarray, $\mathrm{SD}$ - standard deviation.

\section{REFERENCES}

1. Brault L, Gasser C, Bracher F, Huber K, Knapp S, Schwaller J. PIM serine/threonine kinases in the pathogenesis and therapy of hematologic malignancies and solid cancers. Haematologica. 2010; 95:1004-1015.

2. Eichmann A, Yuan L, Breant C, Alitalo K, Koskinen PJ. Developmental expression of pim kinases suggests functions also outside of the hematopoietic system. Oncogene. 2000; 19:1215-1224.

3. Bachmann M, Moroy T. The serine/threonine kinase Pim-1. Int J Biochem Cell Biol. 2005; 37:726-730.

4. Amaravadi R, Thompson CB. The survival kinases Akt and Pim as potential pharmacological targets. J Clin Invest. 2005; 115:2618-2624.

5. Nawijn MC, Alendar A, Berns A. For better or for worse: The role of Pim oncogenes in tumorigenesis. Nat Rev Cancer. 2011; 11:23-34.

6. Wang Z, Bhattacharya N, Weaver M, Petersen K, Meyer M, Gapter L, Magnuson NS. Pim-1: A serine/threonine kinase with a role in cell survival, proliferation, differentiation and tumorigenesis. J Vet Sci. 2001; 2:167-179.

7. Chen J, Kobayashi M, Darmanin S, Qiao Y, Gully C, Zhao R, et al. Hypoxia mediated up-regulation of Pim-1 contributes to solid tumor formation. Am J Pathol. 2009; 175:400-11.

8. Narlik-Grassow M, Blanco-Aparicio C, Cecilia Y, Peregrina S, Serelde BG, Munoz-Galvan S, et al. Essential role of PIM kinases in sarcoma growth and bone invasion. Carcinogenesis. 2012; 33:1479-86.

9. Wen X, Wu JM, Wang FT, Liu B, Huang CH. Free Radical Bio Med!. 2013; 65:402-410.

10. Reiser-Erkan C, Erkan M, Pan Z, Bekasi S, Giese NA, Streit S, et al. Hypoxiainducible proto-oncogene Pim-1 is a prognostic marker in pancreatic ductal adenocarcinoma. Cancer Biol Ther. 2008; 7:1352-9.

11. Morwick T. a survey of the patent literature. Expert Opin Ther Pat. 2010; 20:193-212.

12. Pogacic V, Bullock AN, Fedorov O, Filippakopoulos P, Gasser C, Biondi A, et al. Structural analysis identifies imidazo[1,2-b]pyridazines as PIM kinase inhibitors with in vitro antileukemic activity. Cancer Res. 2007; 67:6916-24.

13. Blanco-Aparicio C, Collazo AM, Oyarzabal J, Leal JF, Albaran MI, Lima FR, et al. Pim 1 kinase inhibitor ETP45299 suppresses cellular proliferation and synergizes with PI3K inhibition. Cancer Lett. 2011; 300:145-53.

14. Carmen BA, Amancio C. Pim kinases in cancer: Diagnostic, prognostic and treatment opportunities. Biochemical Pharmacology. 2013; 85:629-643.

15. Popivanova BK, Li YY, Zheng H, et al. Protooncogene, Pim-3 with serine/threonine kinase activity, is aberrantly expressed in human colon cancer cells and can prevent Badmediated apoptosis. Cancer Sci. 2007; 98:321-328. 
16. Pierre F, Stefan E, Nédellec AS, Chevrel MC, Collin F, Regan, et al. 7-(4H-1,4-Triazol-3-yl)benzoc.2,6.naphthyridines: A novel class of Pim kinase inhibitors with potent cell antiproliferative activity. Bioorganic and Medicinal Chemistry Letters. 2011; 21:6687-6692.

17. Michael P, Dwyera, Kartik K, Kamil P, Carmen A, Marc L, et al. Discovery of pyrazolo1,5-a.pyrimidine-based Pim inhibitors: A template-based approach. Bioorganic and Medicinal Chemistry Letters. 2013; 23:6178-6182.

18. Dakin LA, Block MH, Chen HW, Code E, Dowling JE, Feng XM, et al. Discovery of novel benzylidene-1, 3-thiazolidine-2,4-diones as potent and selective inhibitors of the PIM-1, PIM-2, and PIM-3 protein kinases. Bioorganic and Medicinal Chemistry Letters. 2012; 22:4599-4604.

19. Haddach M, Michaux J, Schwaebe MK, Pierre F, O’Brien SE, Borsan C. Discovery of CX-6258. A potent, selective, and orally efficacious pan-Pim kinases inhibitor. ACS Med Chem Lett. 2012; 3:135-139.

20. Garcia PD, Langowski JL, Wang YY, Chen M, Castillo J, Fanton C. Pan-PIM kinase inhibition provides a novel therapy for treating hematologic cancers. Clin Cancer Res. 2014; 20:1834-1845.

21. Lisa SC, Sanjeev R, David B, William G, Wierda G. Pim kinase inhibitor, SGI-1776, induces apoptosis in chronic lymphocytic leukemia cells. Blood. 2009; 114:4150-4157.

22. Erika KK, Kristen M, Keith SD, Sangeetha P, Cao YC, et al. AZD1208, a potent and selective pan-Pim kinase inhibitor, demonstrates efficacy in preclinical models of acute myeloid leukemia. Blood. 2014; 123:905-913.

23. Mishra GR, Suresh M, Kumaran K, Kannabiran N, Suresh S, Bala P, et al. Human protein reference database2006 update. Nucleic Acids Research. 2006; 34:411-414.

24. Alfarano C, Andrade CE, Anthony K, Bahroos N, Bajec M, Bantoft K, et al. The Biomolecular Interaction Network Database and related tools 2005 update. Nucleic Acids Research. 2005; 33:418-424.

25. Xenarios I, Salwinski L, Duan XJ, Higney P, Kim SM, Eisenberg D. DIP, the Database of Interacting Proteins: a research tool for studying cellular networks of protein interactions. Nucleic Acids Research. 2002; 30:303-305.

26. Mewes HW, Frishman D, Güldener U, Mannhaupt G, Mayer K, Mokrejs M, Morgenstern B, Münsterkötter M, Rudd S, Weil B. MIPS: a database for genomes and protein sequences. Nucleic Acids Research. 2002; 30:31-4.

27. Kerrien S, Alam-Faruque Y, Aranda B, Bancarz I, Bridge A, Derow C, et al. IntAct — open source resource for molecular interaction data. Nucleic Acids Research. 2007; 35:D561-D565.

28. Zhang QC, Petrey D, Garzón JI, Deng L, Honig B. PrePPI: a structure-informed database of protein-protein interactions. Nature. 2012; 490:556-560.

29. Wang N, Xu HL, Zhao X, Wen X, Wang FT, Wang SY, et al. Network-based identification of novel connections among apoptotic signaling pathways in cancer. Applied Biochemistry and Biotechnology. 2012; 167:621-631.

30. Baldi P, Brunak S, Chauvin Y, et al. Assessing the accuracy of prediction algorithms for classification: an overview, Bioinformatics. 2000; 16:412-424.

31. Liu B, Cheng Y, Zhang B, Bian HJ, Bao JK. Polygonatum cyrtonema lectin induces apoptosis and autophagy in human melanoma A375 cells through a mitochondriamediated ROS-p38-p53 pathway. Cancer Lett. 2009; 275:54-60.

32. Mosmann T. Rapid colorimetric assay for cellular growth and survival: application to proliferation and cytotoxicity assays. J Immunol Methods. 1983; 65:55-63.

33. Wei J, Shi J, Zhang J, He G, Pan J, He J, et al. Ouyang, Design, synthesis and biological evaluation of enzymatically cleavable NSAIDs prodrugs derived from self-immolative dendritic scaffolds for the treatment of inflammatory diseases. Bioorg Med Chem. 2013; 21:192-200.

34. Ouyang L, Huang Y, Zhao Y, He G, Xie Y, Liu J, et al. Preparation, antibacterial evaluation and preliminary structure-activity relationship (SAR) study of benzothiazol- and benzoxazol-2-amine derivatives. Bioorg Med Chem Lett. 2012; 22:3044-3049.

35. Pronk S, Páll S, Schulz R, Larsson P, Bjelkmar P, Apostolov R, et al. GROMACS 4.5: a high-throughput and highly parallel open source molecular simulation toolkit. Bioinformatics. 2013; 29:845-854.

36. Zhang T, Xie N, He WF, Liu R, Lei YL, Tan H, et al. An integrated proteomics and bioinformatics analyses of Hepatitis B Virus X protein and identification of a novel interactor apoA-I. Journal of Proteomics. 2013; 84:92-105.

37. Schüttelkopf AW, Van Aalten DM. PRODRG: a tool for high-throughput crystallography of protein-ligand complexes, ActaCrystallogr. D Biol. Crystallogr. 2004; 60:1355-1363.

38. Ouyang L, Chen Y, Wang XY, Lu RF, Zhang SY, Tian M, et al. Polygonatum odoratum lectin induces apoptosis and autophagy via targeting EGFR-mediated RasRaf-MEK-ERK pathway in human MCF-7 breast cells. Phytomedicine. 2014; 21:1658-1665.

39. May MJ, Madge LA. Caspase inhibition sensitizes inhibitor of NFkappaB kinase beta-deficient fibroblasts to caspaseindependent cell death via the generation of reactive oxygen species. J Biol Chem. 2007; 282:16105-16116.

40. Liu B, Wu JM, Li J, Li WW, Liu JJ, Li CY, et al. Polygonatum cyrtonema lectin induces murine fibrosarcoma L9 cell apoptosis and autophagy via blocking RasRaf and PI3K-Akt signaling pathways. Biochimie. 2010; 92:1934-1938.

41. Chen JS, Lin SC, Chen CY, Hsieh YT, Pai PH, Chen LK, et al. Development of a microarray for two rice subspecies: characterization and validation of gene expression in rice tissues. BMC Res. 2014; 8:7-15. 
42. Tusher VG, Tibshirani R, Chu G. Significance analysis of microarrays applied to transcriptional responses to ionizing radiation. Proc Natl Acad Sci. 2001; 98:5116-5121.

43. Tan AC, Naiman DQ, Xu L, et al. Simple decision rules for classifying human cancers from gene expression profiles. Bioinformatics. 2005; 21:3896-3904.

44. Dweep H, Sticht C, Pandey P, Gretz N. miRWalk database: prediction of possible miRNA binding sites by "walking" the genes of 3 genomes. Journal of Biomedical Informatics. 2011; 44:839-837.

45. Kiriakidou M, Nelson PT, Kouranov A, Fitziev P, Bouyioukos C, Mourelatos Z, et al. A combined computational-experimental approach predicts human microRNA targets. Genes Dev. 2004; 18:1165-1178.

46. John B, Enright AJ, Aravin A, Tuschl T, Sander C, Marks DS. Human microRNA targets. PLoS Biol. 2004; 2:e363.

47. Krek A, Grun D, Poy MN, Wolf R, Rosenberg L, Epstein EJ, et al. Combinatorial microRNA target predictions. Nat Genet. 2005; 37:495-500.
48. Wang X. MiRDB: a microRNA target prediction and functional annotation database with a wiki interface. RNA. 2008; 14:1012-1017.

49. Kertesz M, Iovino N, Unnerstall U, Gaul U, Segal E. The role of site accessibility in microRNA target recognition. Nat Genet. 2007; 39:1278-1284.

50. Dweep H, Sticht C, Pandey P, Gretz N. miRWalk-database: prediction of possible miRNA binding sites by "walking" the genes of 3 genomes. Journal of Biomedical Informatics. 2011; 44:839-837.

51. Miranda KC, et al. A pattern-based method for the identification of microRNA binding sites and their corresponding heteroduplexes. Cell. 2006; 126:1203-1217.

52. Rehmsmeier M, Steffen P, Hochsmann M, Giegerich R. Fast and effective prediction of microRNA/target duplexes. RNA. 2014; 10:1507-1517.

53. Lewis BP, Burge CB, Bartel DP. Conserved seed pairing, often flanked by adenosines, indicates that thousands of human genes are microRNA targets. Cell. 2005; 120:5-20. 